\title{
Chemical Mediators: the Remarkable Structure and Host-Selectivity of Depsilairdin, a Sesquiterpenic Depsipeptide Containing a New Amino Acid
}

M. Soledade C. Pedras, ${ }^{*}$ Paulos B. Chumala and J. Wilson Quail

\author{
Corresponding author: M. Soledade C. Pedras \\ Department of Chemistry, University of Saskatchewan, \\ 110 Science Place, Saskatoon SK S7N 5C9, Canada \\ Telephone: +1-306-966-4772 \\ Fax: +1-306-966-4730 \\ E-mail: s.pedras@usask.ca
}




\title{
SUPPLEMENTARY DATA FOR:
}

\section{Chemical Mediators: the Remarkable Structure and Host-Selectivity of Depsilairdin, a Sesquiterpenic Depsipeptide Containing a New}

\author{
Amino Acid \\ M. Soledade C. Pedras,* Paulos B. Chumala and J. Wilson Quail \\ Department of Chemistry, University of Saskatchewan, 110 Science Place, Saskatoon SK \\ S7N 5C9, Canada
}

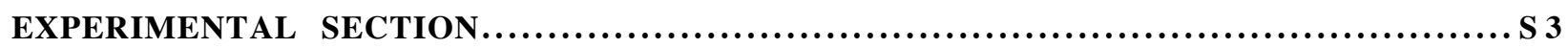

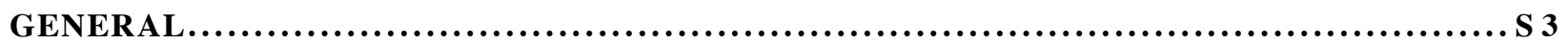

FUNGAL CULTURES AND ISOLATION OF DEPSILAIRDIN.......................... S 3

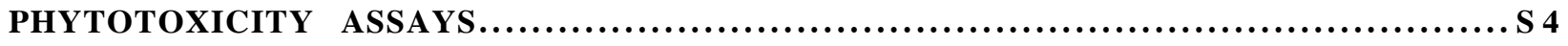

SYNTHESIS OF $(3 S, 6 R)-3,6$-DIISOPROPYL-2,5-MORPHOLIDENONE $(8) \ldots \ldots \ldots \ldots \ldots \ldots . . . . .4$

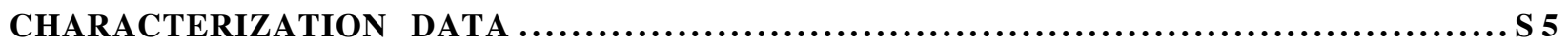

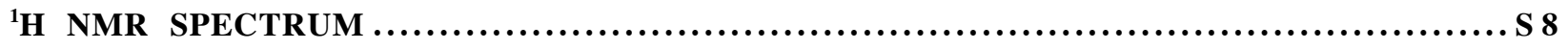

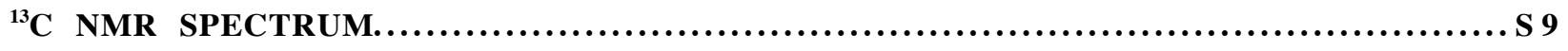

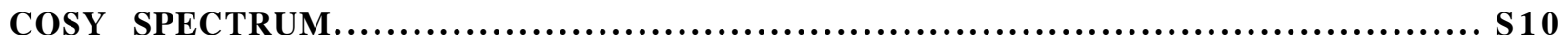

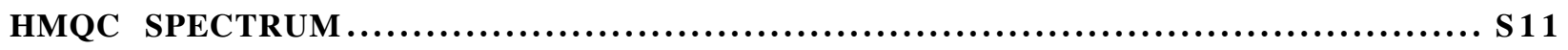

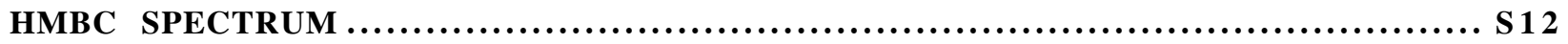


Pedras et al.

Supplementary data

\section{Experimental Section}

\section{General}

All chemicals were purchased from Sigma-Aldrich Canada Ltd., Oakville, ON except for deuterated chemicals and solvents purchased from Cambridge Isotope Laboratories, Inc., Andover, MA. All solvents were HPLC grade and used as such, except for $\mathrm{CH}_{2} \mathrm{Cl}_{2}$ and $\mathrm{CHCl}_{3}$ which were redistilled and THF which was dried over Na and benzophenone. Flash CC: JT Baker WP C-18 reversed phase silica gel $40 \mu \mathrm{m}$. Analytical HPLC analysis was carried out with a high performance liquid chromatograph equipped with quaternary pump, automatic injector, and photodiode array detector (wavelength range 190-600 nm), degasser, and a Hypersil ODS column (5 $\mu \mathrm{m}$ particle size silica, 4.6 i.d. $\times 200 \mathrm{~mm}$ ), equipped with an in-line filter. Mobile phase: $\mathrm{H}_{2} \mathrm{O}-\mathrm{CH}_{3} \mathrm{CN}$ 20:80, 10 min, gradient 20:80 to $60: 40,10 \mathrm{~min}, 60: 40$ to $75-25,10 \mathrm{~min}, 75-25$ to $100 \mathrm{CH}_{3} \mathrm{CN}, 10 \mathrm{~min}$, and flow rate $1.0 \mathrm{~mL} / \mathrm{min}$.

\section{Fungal Cultures and Isolation of Depsilairdin}

Isolates of L. maculans/P. lingam (Mayfair 2 and Laird 2) solid cultures (to provide spores to inoculate liquid cultures) were grown on V8 agar medium, and liquid cultures were grown in 250 mL Erlenmeyer flasks containing $100 \mathrm{~mL}$ of a chemically defined medium, as previously reported. ${ }^{1}$ Liquid cultures (122 L) were incubated for six days, were filtered, the broth was concentrated to 22 L by freeze drying, and then was extracted with ethyl acetate. The combined extracts were dried over anhydrous sodium sulfate and concentrated to dryness under reduced pressure. The ethyl acetate extracts (ca. $350 \mathrm{mg}$ per batch, total amount $2.3 \mathrm{~g}$ ) were subjected to flash column chromatography (silica gel, $2 \mathrm{~cm} \times 15 \mathrm{~cm}, 50 \mathrm{ml}$ fractions, $\mathrm{CH}_{2} \mathrm{Cl}_{2}, 200 \mathrm{~mL}$ followed by $\mathrm{CH}_{2} \mathrm{Cl}_{2}-\mathrm{MeOH}, 97: 3,200$ $\mathrm{mL}$, and then $\mathrm{CH}_{2} \mathrm{Cl}_{2}-\mathrm{MeOH}, 90: 10,200 \mathrm{~mL}$ ). Fractions were concentrated to dryness under reduced pressure. The most phytotoxic fractions $\left(\mathrm{F}_{8}-\mathrm{F}_{10}\right)$ were combined and subjected to further

\footnotetext{
${ }^{1}$ Pedras, M. S. C.; Biesenthal, C. J. Phytochemistry 2001, 58, 905-909.
} 
chromatography $\left(\mathrm{C}_{18}\right.$ column and prep. TLC) to yield depsilairdin (7) $(22 \mathrm{mg}$, ca. $0.2 \mathrm{mg} / \mathrm{L}$ of culture).

\section{Phytotoxicity Assays}

The selective phytotoxicity of depsilairdin (7) to plants resistant and susceptible to blackleg isolates Mayfair 2 and Laird 2 (canola, resistant; brown mustard, susceptible; white mustard, tolerant) was evaluated as previously reported for other toxins. ${ }^{2}$ Seeds of canola (B. napus) cv. Westar, white mustard (Sinapis alba) cv. Ochre, and brown mustard (B. juncea) cv. Cutlass were obtained from Plant Gene Resources, Agriculture and Agric-Food Canada Research Station, Saskatoon, SK. Plants were grown in a growth chamber, under controlled environmental conditions, as previously reported. ${ }^{2}$ Phytotoxicity assays were conducted on leaves of two-week-old plants with depsilairdin (7) $\left(1.0 \times 10^{-3}-5.0 \times 10^{-6} \mathrm{M}\right.$ in water-methanol, $\left.1: 1, \mathrm{v} / \mathrm{v}\right)$, as previously described for other compounds. ${ }^{2}$ Plants were incubated in a growth chamber for seven days and visual symptoms recorded.

\section{Synthesis of (3S,6R)-3,6-diisopropyl-2,5-morpholinedione (8)}

A solution of $N, N^{\prime}$-carbonyldiimidazole (CDI) ( $\left.67 \mathrm{mg}, 0.4 \mathrm{mmol}\right)$ in THF (1 mL) was added to a solution of $N$-Me-Cbz-Val $(9,110 \mathrm{mg}, 0.4 \mathrm{mmol})$ in THF (1 mL) and the solution was stirred under argon atmosphere at room temperature for $4 \mathrm{~h}$. $(R)$-2-Hydroxy-3-methylbutyric acid (10, 24 $\mathrm{mg}, 0.2 \mathrm{mmol})$ in THF (1 mL) was added to the reaction mixture, the mixture was stirred for another $30 \mathrm{~h}$ at room temperature, and the solvent was removed in vacuum. After chromatography, the product (54 mg, $0.15 \mathrm{mmol}$ ) was dissolved in $2 \mathrm{~mL}$ of methanol, followed by addition of $10 \%$ $\mathrm{Pd} / \mathrm{C}(12 \mathrm{mg})$ and the mixture was vigorously stirred under an $\mathrm{H}_{2}$ atmosphere (balloon) for $2 \mathrm{~h}$. Then the mixture was filtered through Celite and the combined filtrate and washings were concentrated to yield the deprotected didepsipeptide 11 quantitatively. Didepsipeptide 11 (16 mg,

\footnotetext{
${ }^{2}$ Pedras, M. S. C.; Biesenthal, C.J.; Zaharia, I. L. Plant Science 2000, 156, 185-192.
} 
$0.06 \mathrm{mmol}$ ) was suspended in $\mathrm{CH}_{2} \mathrm{Cl}_{2}(4 \mathrm{~mL})$ and treated with 2-chloro-1-methylpyridinium iodide (26 mg, $0.10 \mathrm{mmol})$. After addition of triethylamine $(24 \mu \mathrm{L}, 0.18 \mathrm{mmol})$, the reaction mixture was kept under reflux for $4 \mathrm{~h}$. Finally the solvent was removed in vacuum and the residue was chromatographed to yield $\mathbf{8}$ in $69 \%$ overall yield.

\section{Characterization data}

Depsilairdin (7). HPLC $\mathrm{R}_{\mathrm{t}}=28.4 \mathrm{~min} .[\alpha]_{\mathrm{D}}=-65\left(\mathrm{c} 0.90, \mathrm{CH}_{2} \mathrm{Cl}_{2}\right) .{ }^{1} \mathrm{H}$ NMR in Table $;{ }^{13} \mathrm{C}$ NMR in Table; FTIR (cm $\left.{ }^{-1}\right): 3449,2962,1635,1455,1200,1136$. UV $\lambda_{\max }(\log \varepsilon) 207$ (3.6). HREI-MS: $m / z$ 707.4724, calc. for $\mathrm{C}_{38} \mathrm{H}_{65} \mathrm{~N}_{3} \mathrm{O}_{9}$ 707.4721. EI-MS: $m / z$ (relative intensity) 707 (11\%), 634 (50\%), $578(45 \%), 521(39 \%) 465(100 \%)$.

Table. ${ }^{1} \mathrm{H}$ NMR (500 MHz), ${ }^{13} \mathrm{C}$ NMR (500 MHz), and HMBC data of depsilairdin (7) in $\mathrm{C}_{6} \mathrm{D}_{6}$.

\begin{tabular}{llll}
\hline Carbon & $\delta_{\mathrm{C}}(\mathrm{ppm})$ & $\delta_{\mathrm{H}}(\mathrm{ppm}), J(\mathrm{~Hz})$ & $\mathrm{HMBC}$ \\
\hline 1 & 171.7 & - & - \\
2 & 67.8 & $4.47, \mathrm{br} \mathrm{s}$ & $1,3,5,6$ \\
3 & 76.6 & - & - \\
4 & 76.4 & $4.47, \mathrm{~m}$ & 3 \\
5 & 52.8 & $4.48, \mathrm{~m}$ & 2,4 \\
& & $3.89, \mathrm{dd}, 12,4$ & 4 \\
6 & 24.8 & $1.28, \mathrm{~s}$ & $2,3,4$ \\
7 & 170.1 & - & - \\
8 & 59.4 & $5.29, \mathrm{~d}, 11$ & $7,9,11,12,13$ \\
9 & 28.0 & $2.47, \mathrm{~m}$ & $8,10,11$ \\
10 & 20.3 & $1.18, \mathrm{~d}, 7$ & $8,9,11$ \\
11 & 18.8 & $0.83, \mathrm{~d}, 7 \mathrm{~Hz}$ & $8,9,10$ \\
12 & 31.0 & $3.36, \mathrm{~s}$ & 8,13 \\
13 & 171.1 & - & - \\
14 & 59.5 & $5.34, \mathrm{~d}, 11 \mathrm{~Hz}$ & 13,15 \\
15 & 27.8 & $2.45, \mathrm{~m}$ & $14,16.17$ \\
16 & 19.5 & $0.92, \mathrm{~d} 7 \mathrm{~Hz}$ & $14,15,17$ \\
17 & 18.7 & $0.81, \mathrm{~d} 7 \mathrm{~Hz}$ & $14,15,16$ \\
18 & 29.8 & $2.74, \mathrm{~s}$ & 14,19 \\
19 & 175.4 & - & - \\
20 & 72.6 & $4.22, \mathrm{br} \mathrm{d}, 6$ & $19,21,22,23$ \\
21 & 31.0 & $1.80, \mathrm{~m}$ & 22,23 \\
\hline
\end{tabular}




\begin{tabular}{llll}
\hline 22 & 19.5 & $1.18, \mathrm{~d}, 7 \mathrm{~Hz}$ & $20,21,23$ \\
23 & 15.0 & $0.99, \mathrm{~d}, 7$ & $20,21,22$ \\
$1^{\prime}$ & 83.3 & $4.89, \mathrm{dd}, 12,4$ & $2^{\prime}, 9^{\prime}, 10^{\prime}, 15^{\prime}, 1$ \\
$2^{\prime}$ & 25.8 & $2.10, \mathrm{~m} ; 1.78, \mathrm{~m}$ & $1^{\prime}, 3^{\prime}, 4^{\prime}$ \\
$3^{\prime}$ & 41.1 & $1.64, \mathrm{~m} ; 1.44, \mathrm{~m}$ & $1^{\prime}, 2^{\prime}, 4^{\prime}, 5^{\prime}$ \\
$4^{\prime}$ & 70.7 & - & - \\
$5^{\prime}$ & 53.5 & $1.26, \mathrm{~m}$ & $4^{\prime}, 6^{\prime}, 7^{\prime}, 10^{\prime}, 15^{\prime}$ \\
$6^{\prime}$ & 26.1 & $2.08, \mathrm{~m} ; 1.28, \mathrm{~m}$ & $5^{\prime}, 7^{\prime}, 10^{\prime}$ \\
$7^{\prime}$ & 46.1 & $1.94, \mathrm{~m}$ & $6^{\prime}$ \\
$8^{\prime}$ & 26.9 & $1.62, \mathrm{~m} ; 1.41, \mathrm{~m}$ & $1^{\prime}$ \\
$9^{\prime}$ & 40.7 & $1.19, \mathrm{~m} ; 2.05, \mathrm{~m}$ & $5^{\prime}, 8^{\prime}, 10^{\prime}$ \\
$10^{\prime}$ & 38.9 & - & - \\
$12^{\prime}$ & 108.4 & $4.92, \mathrm{~s} ; 4.93, \mathrm{~s}$ & $7^{\prime}, 13^{\prime}$ \\
$11^{\prime}$ & 150.1 & - & - \\
$13^{\prime}$ & 21.3 & $1.80, \mathrm{~s}$ & $7^{\prime}, 11^{\prime}, 12^{\prime}$ \\
$14^{\prime}$ & 22.7 & $1.00, \mathrm{~s}$ & $3^{\prime}, 4^{\prime}, 5^{\prime}$ \\
$15^{\prime}$ & 14.5 & $1.09, \mathrm{~s}$ & $1^{\prime}, 5^{\prime}, 9^{\prime}, 10^{\prime}$ \\
\hline
\end{tabular}

(3S,6R)-3,6-Diisopropyl-2,5-morpholinedione (8). HPLC $\mathrm{R}_{\mathrm{t}}=19.9$ min. $[\alpha]_{\mathrm{D}}=168$ (c 0.94 , $\left.\mathrm{CH}_{2} \mathrm{Cl}_{2}\right) ;{ }^{1} \mathrm{H} \mathrm{NMR}\left(\mathrm{CDCl}_{3}\right): \delta_{\mathrm{H}} 0.94(\mathrm{~d}, J=7 \mathrm{~Hz}), 1.07(\mathrm{~d}, J=7 \mathrm{~Hz}), 1.14(\mathrm{~d}, J=7 \mathrm{~Hz}), 1.17(\mathrm{~d}, J$ $=7 \mathrm{~Hz}), 2.30(\mathrm{~m}), 2.64(\mathrm{~m}), 3.05(\mathrm{~s}), 3.87(\mathrm{~d}, J=6 \mathrm{~Hz}), 4.73(\mathrm{~d}, J=2 \mathrm{~Hz}) ;{ }^{13} \mathrm{C} \mathrm{NMR}\left(\mathrm{CDCl}_{3}\right): \delta_{\mathrm{C}}$ 15.7, 18.6, 19.2, 20.0, 30.8, 32.2, 34.7, 67.8, 82.3, 165.9, 166.3. HREI-MS: $m / z$ 213.1364, calc. for $\mathrm{C}_{11} \mathrm{H}_{19} \mathrm{O}_{3} \mathrm{~N}$ 213.1365, EI-MS: $\mathrm{m} / z$ (relative intensity) 213 (8\%), 171 (100\%).

(3R,6R)-3,6-Diisopropyl-2,5-morpholinedione (14). HPLC $\mathrm{R}_{\mathrm{t}}=20.1$ min. $[\alpha]_{\mathrm{D}}=31$ (c 0.95 , $\left.\mathrm{CH}_{2} \mathrm{Cl}_{2}\right)^{1} \mathrm{H} \mathrm{NMR}\left(\mathrm{CDCl}_{3}\right): \delta_{\mathrm{H}} 1.06(\mathrm{~d}, J=7 \mathrm{~Hz}), 1.08(\mathrm{~d}, J=7 \mathrm{~Hz}), 1.15(\mathrm{~d}, J=7 \mathrm{~Hz}), 1.2(\mathrm{~d}, J=$ $7 \mathrm{~Hz}), 2.30(\mathrm{~m}), 2.33(\mathrm{~m}), 3.04(\mathrm{~s}), 3.85(\mathrm{~d}, J=6 \mathrm{~Hz}), 4.53(\mathrm{~d}, J=6 \mathrm{~Hz}) ;{ }^{13} \mathrm{C} \mathrm{NMR} \quad\left(\mathrm{CDCl}_{3}\right): \delta_{\mathrm{C}}$ 18.6, 19.4, 20.0, 20.4, 32.7, 32.8, 34.7, 66.8, 84.0, 165.4, 165.7. HREI-MS: $\mathrm{m} / \mathrm{z}$ 213.1371, calc. for $\mathrm{C}_{11} \mathrm{H}_{19} \mathrm{O}_{3} \mathrm{~N} 213.1365$, EI-MS: $\mathrm{m} / z$ (relative intensity) 213 (12\%), 171 (100\%).

(3R,6S)-3,6-Diisopropyl-2,5-morpholinedione (17). HPLC $R_{t}=19.9$ min. $[\alpha]_{D}=-179(\mathrm{c} 1.15$, $\left.\mathrm{CH}_{2} \mathrm{Cl}_{2}\right) .{ }^{1} \mathrm{H} \mathrm{NMR}\left(\mathrm{CDCl}_{3}\right): \delta_{\mathrm{H}} 0.94(\mathrm{~d}, J=7 \mathrm{~Hz}), 1.07(\mathrm{~d}, J=7 \mathrm{~Hz}), 1.14(\mathrm{~d}, J=7 \mathrm{~Hz}), 1.17(\mathrm{~d}, J$ $=7 \mathrm{~Hz}), 2.30(\mathrm{~m}), 2.64(\mathrm{~m}), 3.05(\mathrm{~s}), 3.87(\mathrm{~d}, J=6 \mathrm{~Hz}), 4.73(\mathrm{~d}, J=2 \mathrm{~Hz}) \cdot{ }^{13} \mathrm{C} \mathrm{NMR}\left(\mathrm{CDCl}_{3}\right): \delta_{\mathrm{C}}$ 
Pedras et al.

Supplementary data

15.7, 18.6, 19.2 , 20.0, 30.8, 32.2, 34.7, 67.8), 82.3, 165.9, 166.3. HREI-MS: $m / z$ 213.1364, calc. for $\mathrm{C}_{11} \mathrm{H}_{19} \mathrm{O}_{3} \mathrm{~N}$ 213.1365, EI-MS: $m / z$ (relative intensity) 213 (8\%), 171 (100\%). 
Pedras et al.

Supplementary data

\section{${ }^{1}$ H NMR Spectrum}

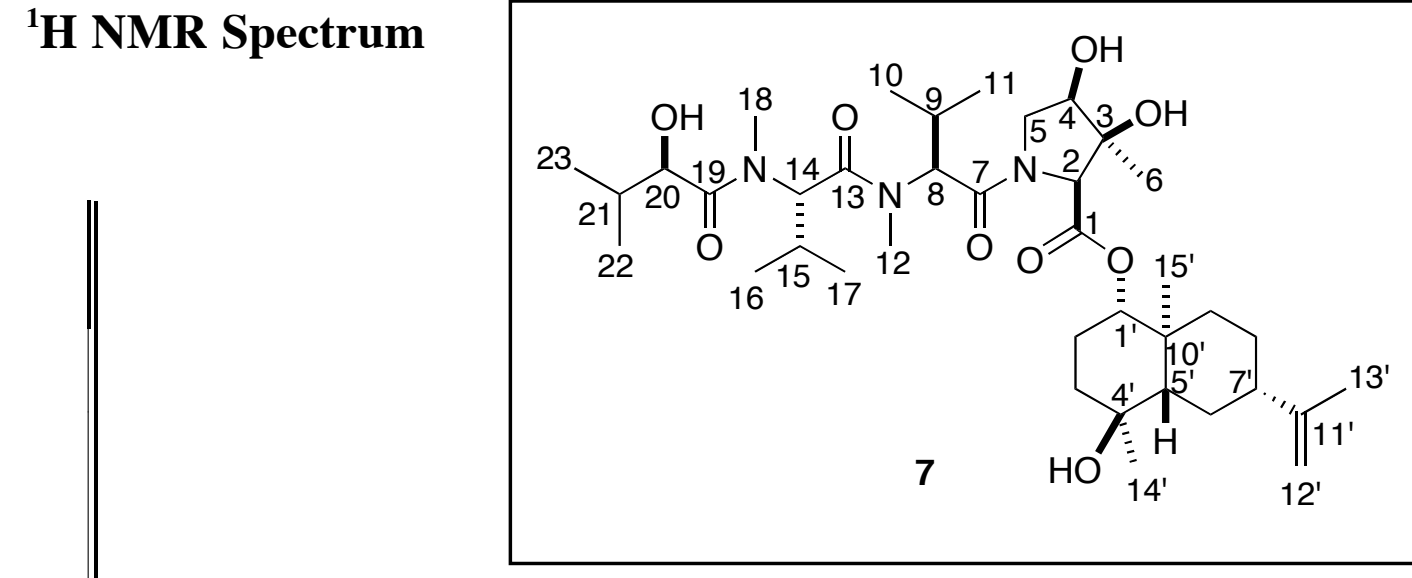


Pedras et al.

Supplementary data

\section{${ }^{13}$ C NMR Spectrum}

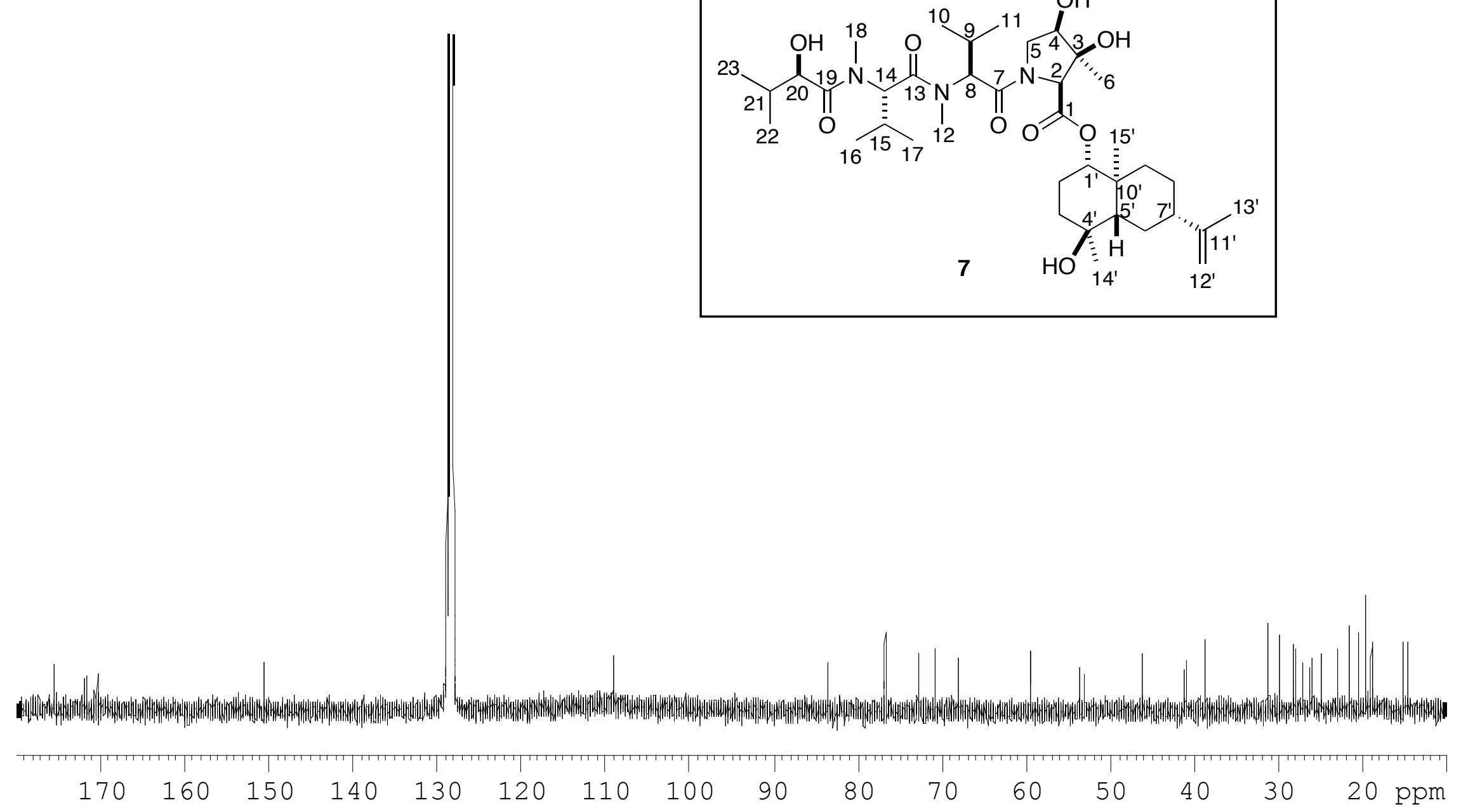


Pedras et al.

Supplementary data

\section{COSY Spectrum}

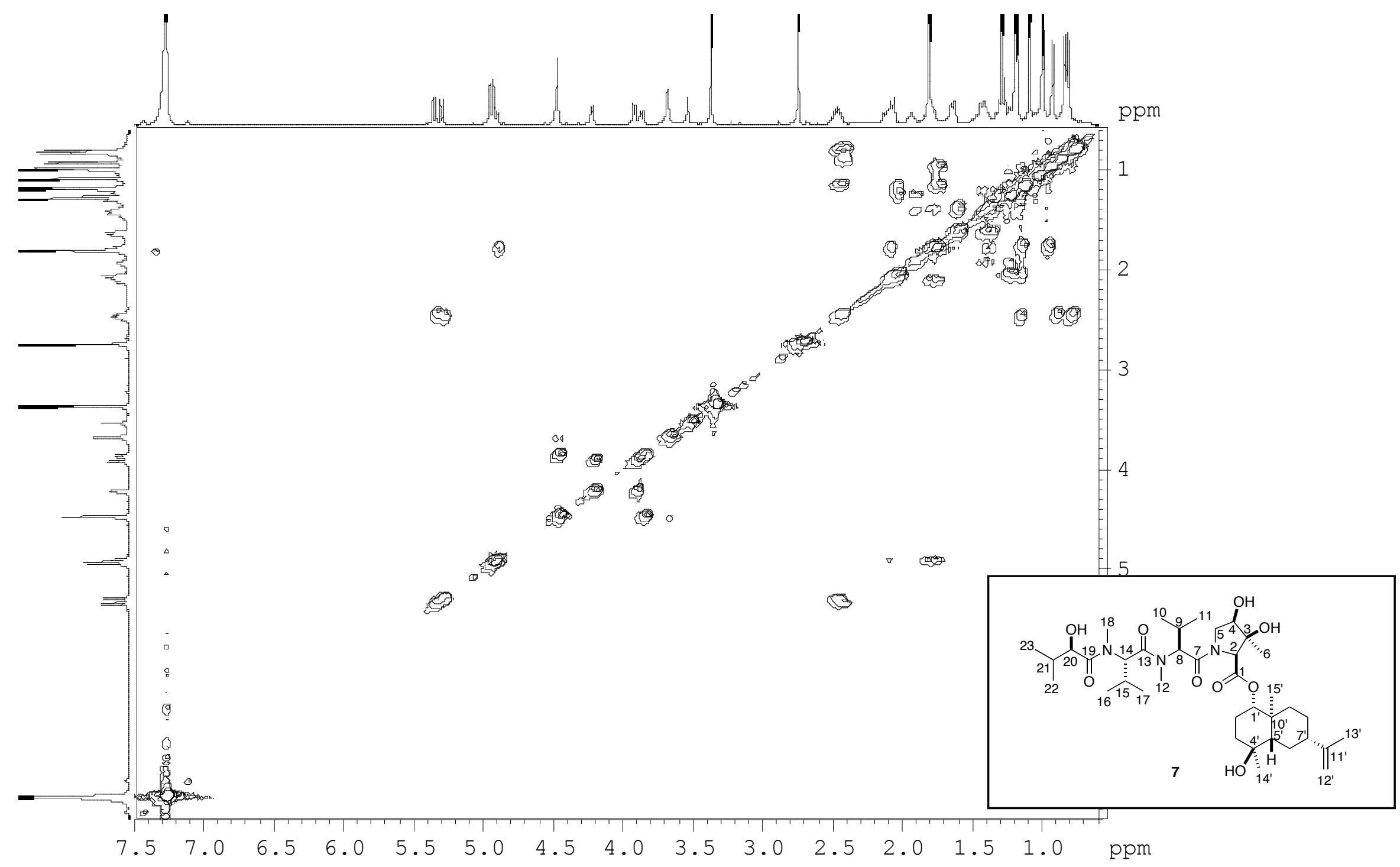


Pedras et al.

Supplementary data

\section{HMQC Spectrum}

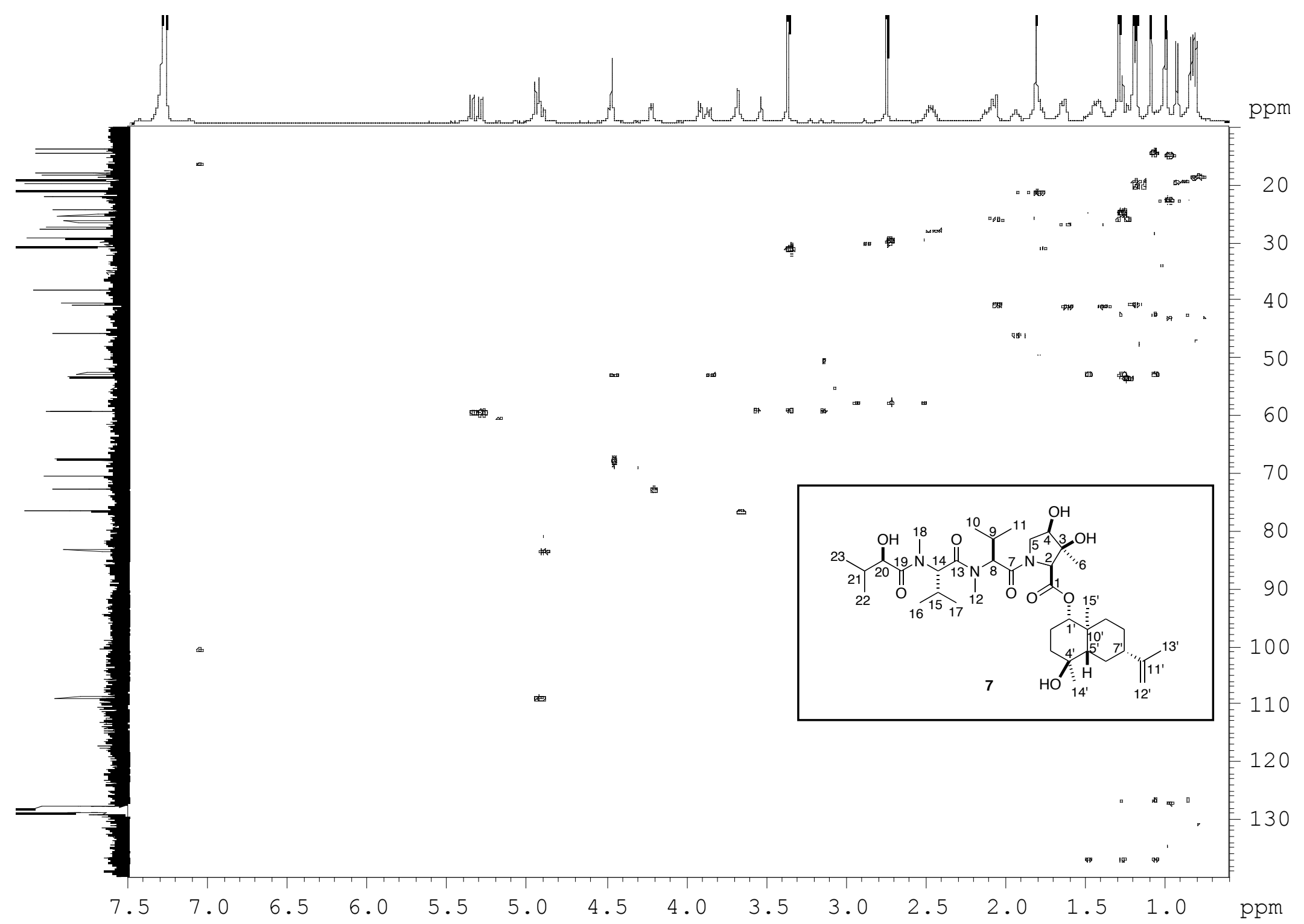


Pedras et al.

Supplementary data

\section{HMBC Spectrum}

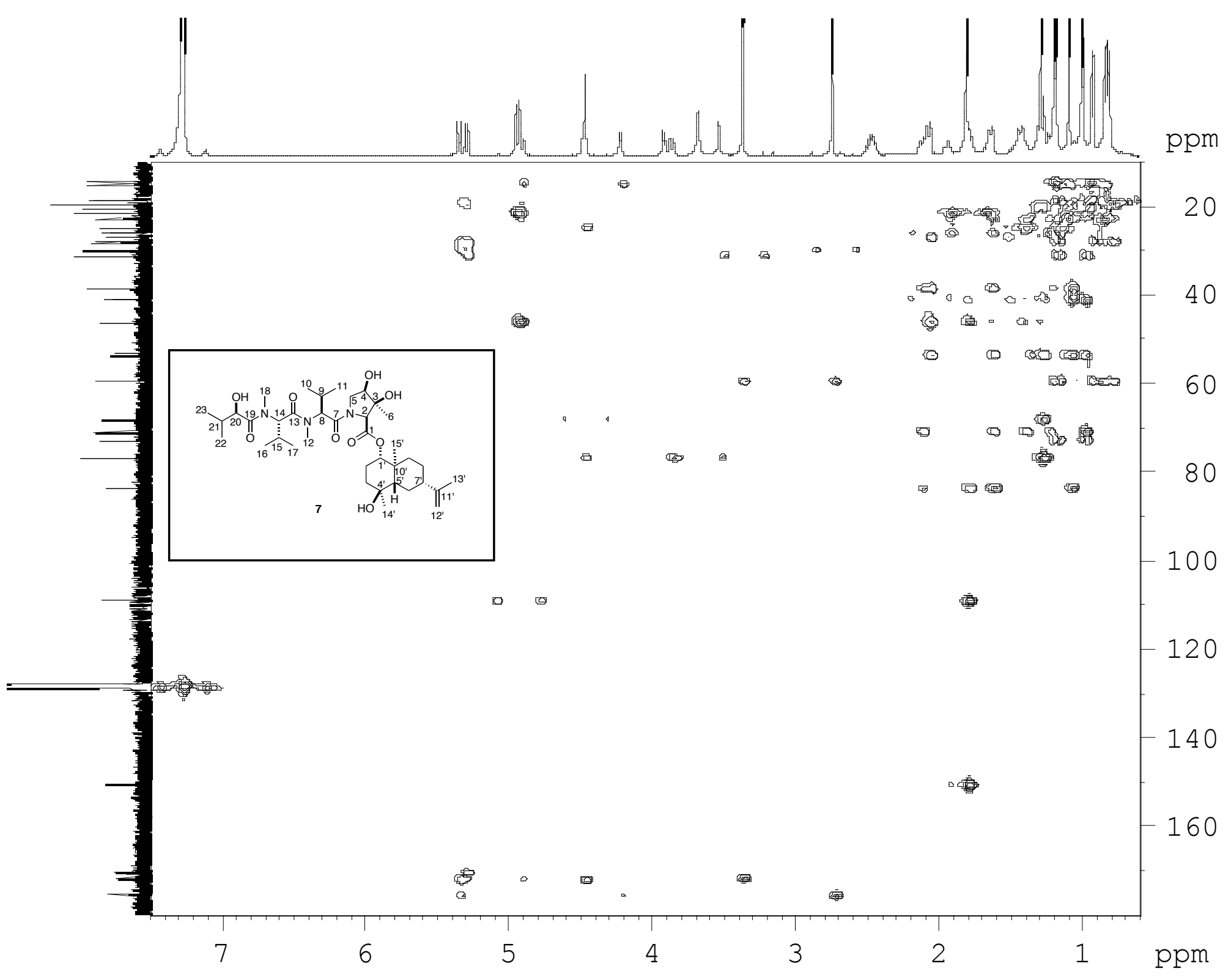

